\title{
Structural composition of components of geoherb Moutan Cortex contributes to anti-diabetic nephropathy activity
}

\author{
Liang Feng ${ }^{1,3,4, *}$, Mao-Mao Zhu' ${ }^{2, *}$, Jun-Fei Gu ${ }^{1,3, *}$, Ming-Hua Zhang ${ }^{1,4,5}$, Chun-Fei \\ Wang $^{1}$, Gang Wang ${ }^{1}$, Jie Song ${ }^{1}$, Xiaobin Tan ${ }^{1}$, Yuanli Zhou ${ }^{1}$, Juan Chen ${ }^{1,4}$, Rong Li ${ }^{2}$, \\ Ling Qiao², Jia Song ${ }^{2}$, Chaochao Wang ${ }^{2}$, Li Zhang ${ }^{2}$ and Xiao-Bin Jiaa ${ }^{1,4}$ \\ ${ }^{1}$ Key Laboratory of New Drug Delivery Systems of Chinese Materia Medica, Jiangsu Provincial Academy of Chinese Medicine, \\ Nanjing 210028, China \\ ${ }^{2}$ Nanjing Institute of Product Quality Inspection, Nanjing 210028, China \\ ${ }^{3}$ State Key Laboratory Breeding Base of Dao-di Herbs, China Academy of Chinese Medical Sciences, Beijing 100700, China \\ ${ }^{4}$ College of Pharmacy, Nanjing University of Chinese Medicine, Nanjing 210023, China \\ ${ }^{5}$ Wuxi Xishan People's Hospital, Wuxi 214011, China \\ *These authors contributed equally to this work
}

Correspondence to: Liang Feng, email: wenmoxiushi@163.com

Xiao-Bin Jia, email: jiaxiaobin2015@163.com

Keywords: Moutan Cortex; structural composition of components; geoherb; anti-diabetic nephropathy; HBZY-1 cells

Received: October 10, 2017

Accepted: December 05, 2017

Published: January 03, 2018

Copyright: Feng et al. This is an open-access article distributed under the terms of the Creative Commons Attribution License 3.0 (CC BY 3.0), which permits unrestricted use, distribution, and reproduction in any medium, provided the original author and source are credited.

\section{ABSTRACT}

Moutan Cortex (MC), a well-known medicinal herb and distributed in multiple regions of China, has been found to be benefical for improving diabetic nephropathy (DN). The geoherb one worked particularly well with specific structural composition of components (SCC). However, whether its better efficacy of geoherb MC than others on DN was attributed to SCC was still unclear. Medicinal plants of MC were collected from ten regions of China, including Gansu, Chongqing, Shandong, Sichuan, Zhejiang, Hunan, Guizhou, Hebei, Henan and Anhui provinces, which were classified for three categories by cluster analysis. HPLC analysis demonstrated that the content and structural composition of 13 compounds were discrepancy. Principal component analysis (PCA) showed six compounds including oxypaeoniflorin, paeoniflorin, trigalloyl glucose, pentagalloyl glucose, benzoylpaeoniflorin and paeonol were the main contributors to its anti-DN activity via increasing superoxide dismutase (SOD) activity, decreasing malondialdehyde (MDA) content, reducing IL-6 and MCP-1 levels in vitro. The results of advanced glycation end products (AGEs)-induced HBZY-1 cells and STZ-induced DN rats showed a significant efficacy difference among MC from ten regions on catalase (CAT), glutathione peroxidase (GSH-Px) activities, intercellular adhesion molecule-1 (ICAM-1), transforming growth factor- $\beta 1$ (TGF- $\beta 1$ ) and fibronectin (FN) protein expression in vivo and in vitro. The recombination of SCC in Hebei MC (worst efficacy) to Anhui MC (best efficacy) showed a similar efficacy. The results demonstrated that the efficacy of MC was closely related to its SCC. Our findings provide evidence for the importance of SCC in quality control about Chinese materia medica and also provide an novel insight into the better efficacy of geoherb one.

\section{INTRODUCTION}

The quality of Chinese materia medica (CMM) is a key factor to ensure the efficacy. The quality inconsistency of CMM from different regions has been found to be closely related to non-repeatability of clinical efficacy and safety [1].
However, the reason why the inconsistency in efficacy and safety of CMM from different regions is not only associated with contained compounds, but also with structural composition of components (SCC). The stable SCC acts as a crucial contributor to the quality consistency of CMM which is related to the better efficacy and monitored safety. 
Geoherbs, the famous authentic herbs, which grew under the specific natural conditions and ecological environment, play an indispensable role in the prevention and treatment of diseases in clinic [2]. Geoherbs had been considered as the best quality of CMM with better efficay than others [3]. The best quality with better function is caused by the special and stable proportion of active components, namely SCC, not the unique compounds or higher content of some compounds in geoherbs. Therefore, the structural proportion of these components could not only reveal the constitutive characteristics of CMM, but also provide effective strategy for the quality control of CMM.

Moutan Cortex (MC), the dried root bark of dicotyledonous plants Paeonia suffruticosa Andr., has a significant efficacy on detoxification and blood circulation [4, 5]. MC, recorded in the "Shen Nong's Herbal Classic", was used for the treatment of cardiovascular disease, hepatoprotective effect, hypoglycemic, hypolipidemic, hypotensive and the central nervous system [6-8]. Additionally, our previous research has confirmed the attenuation of $\mathrm{MC}$ on oxidative stress for renal injury in AGEs-induced mesangial cells dysfunction and streptozotocin-induced $\mathrm{DN}$ rats [9-11]. $\mathrm{MC}$, distributed in multiple regions of China, has a good protective effect on DN, especially the geoherb one. There were great differences between SCC and efficacy in $\mathrm{MC}$ from different regions. To reveal the better efficacy of geoherb $\mathrm{MC}$ and provide further experimental support for quality control of CMM, the present study was designed to compare the difference on SCC and efficacy of MC between geoherb and nongeoherb and also clarified the SCC related to the active components in geoherbs.

\section{RESULTS}

\section{Comparison of MC from different regions on CAT and GSH-Px activities in AGEs-induced HBZY-1 mesangial cells}

As shown in Table 1, the CAT $(2.29 \pm 0.21 \mathrm{U} / \mathrm{mL})$ and GSH-Px (204.17 $\pm 37.10 \mathrm{U} / \mathrm{L})$ activities in HBZY-1 mesangial cells were decreased remarkably by $200 \mu \mathrm{g} / \mathrm{mL}$ AGEs, compared with blank control group $(P<0.001)$. The treatment of MC from different regions could improve AGEs-decreased CAT and GSH-Px activities significantly $(P<0.001, P<0.01)$. Interestingly, Anhui MC, geoherb one, was considered to have the best activity on improving CAT $(6.81 \pm 0.42 \mathrm{U} / \mathrm{mL})$ and GSH-Px (413.44 $\pm 8.45 \mathrm{U} / \mathrm{L})$ activities. The results demonstrated that $\mathrm{MC}$ from different regions made different contributions to attenuate AGEsinduced oxidative stress. Especially, the geoherb MC (Anhui) held the better activity on improving the activities of CAT and GSH-Px than others.

\section{Difference of MC on ICAM-1, TGF- $\beta 1$ and FN expressions in AGEs-induced HBZY-1 mesangial cells}

Western blotting was used to evaluate the protein expressions of ICAM-1, TGF- $\beta 1$ and FN after HBZY1 mesangial cells exposed to AGEs in the presence of $\mathrm{MC}$ from different regions. As shown in Figure 1A, the stimulation of AGEs could increase significantly these protein expressions. The treatment of $\mathrm{MC}$ from different regions could significantly decrease the levels of inflammatory factors in AGEs-induced mesangial cells. The pharmacological order of $\mathrm{MC}$ from different regions on decreasing ICAM-1 expression from high to low was Anhui, Guizhou, Zhejiang, Henan, Shandong, Hunan, Hebei, Sichuan, Gansu and Chongqing (Figure 1B). Similarly, the order of MC to decrease the TGF- $\beta 1$ expression from high to low was Anhui, Sichuan, Shandong, Gansu, Chongqing, Hunan, Henan, Zhejiang, Guizhou and Hebei (Figure 1C). The results shown in Figure 1D indicated that the pharmacological order of MC decreasing FN expression from high to low were Shandong, Anhui, Sichuan, Guizhou, Zhejiang, Gansu, Hunan and Hebei. Generally, Anhui MC, geoherb one, was considered as the best to decrease ICAM1 , TGF- $\beta 1$ and FN expressions.

\section{Effects of MC from different regions on CAT and GSH-Px activities in DN rats}

After the treatment of $\mathrm{MC}$ from different regions, CAT and GSH-Px activities in serum of DN rats were shown in Table 2. CAT and GSH-Px activities of control group were $5.42 \pm 0.410 \mathrm{U} / \mathrm{mL}$ and $458.01 \pm 39.112 \mathrm{U} / \mathrm{L}$, respectively. However, these activities in model group were reduced significantly (CAT: $P<0.01$, GSH-Px: $P<$ 0.001). Compared with the model group, their activities were increased in MC-treated groups and positive drug (AG) group. The ability to improve CAT activity from high to low was Anhui, Sichuan, Hunan, Guizhou, Chongqing, Gansu, Zhejiang, Shandong, Henan and Hebei; while the ability to increase GSH-Px activity from high to low was as follow: Anhui, Shandong, Henan, Zhejiang, Chongqing, Sichuan, Hebei, Hunan, Guizhou and Gansu. The results also showed that Anhui MC had the best efficacy on increasing CAT and GSH-Px activities in vivo.

\section{Reduction of MC on ICAM-1 and TGF- $\beta 1$ expressions in vivo}

As shown in Figure 2, there were no obvious pathological changes in kidney tissues of control group. Namely, ICAM- 1 and TGF- $\beta 1$ expressions were observed normal under light microscope. In DN model group, ICAM-1 and TGF- $\beta 1$ expressions were significantly increased compared with control group. When DN rats were treated with $\mathrm{MC}$ from 10 regions and $\mathrm{AG}$, the relative 
Table 1: Effect of Moutan Cortex from different regions on AGEs-induced CAT and GSH-Px activities $(n=6)$

\begin{tabular}{|c|c|c|}
\hline NO. & $\mathrm{CAT}(\mathrm{U} / \mathrm{mL})$ & GSH-Px (U/L) \\
\hline BSA & $4.34 \pm 0.43$ & $329.44 \pm 29.55$ \\
\hline AGEs & $2.29 \pm 0.21^{\# \#}$ & $204.17 \pm 37.10^{\# \# \#}$ \\
\hline $\mathrm{AGEs}+\mathrm{AG}$ & $5.48 \pm 0.15^{* * *}$ & $337.92 \pm 34.46^{* * *}$ \\
\hline AGEs + Anhui MC & $6.81 \pm 0.42^{* * *}$ & $413.44 \pm 8.45^{* * *}$ \\
\hline AGEs + Guizhou MC & $5.88 \pm 0.11^{* * * \$ s}$ & $255.93 \pm 55.5^{\$ \$ S}$ \\
\hline AGEs + Zhejiang MC & $6.27 \pm 0.15^{* * * s}$ & $353.98 \pm 21.53^{* * * \$}$ \\
\hline AGEs + Henan MC & $5.55 \pm 0.07^{* * * \$ s}$ & $272.17 \pm 19.35^{* \$ s s}$ \\
\hline AGEs + Hunan MC & $5.57 \pm 0.26^{* * * \$ \$}$ & $307.57 \pm 30.15^{* \$ \$}$ \\
\hline AGEs + HebeiMC & $4.52 \pm 0.18^{* * * \$ \$ \$}$ & $236.02 \pm 7.74^{\mathrm{sss}}$ \\
\hline AGEs + Sichuan MC & $6.47 \pm 0.41^{* * *}$ & $318.91 \pm 33.53^{* * \$ \$ \$}$ \\
\hline AGEs + Chongqing MC & $6.12 \pm 0.32^{* * * s}$ & $295.09 \pm 23.78^{* \$ \$ \$}$ \\
\hline AGEs + Shandong MC & $5.46 \pm 0.11^{* * * \$ \$}$ & $371.37 \pm 37.33^{* * *}$ \\
\hline AGEs + Gansu MC & $4.89 \pm 0.46^{* * *} \$ s s$ & $297.03 \pm 32.17^{* \$ \$ s}$ \\
\hline
\end{tabular}

Note: ${ }^{\# \#} P<0.001$ vs. BSA group; ${ }^{*} P<0.05,{ }^{* *} P<0.01$ and ${ }^{* * *} P<0.001$ vs. AGEs group; ${ }^{\$} P<0.05,{ }^{\$ \$} P<0.01$ and ${ }^{\$ \$ \$} P<$ 0.001 vs. Anhui MC group.

expression levels of these inflammation cytokines ICAM1 and TGF- $\beta 1$ in DN rats was relived significantly. Figure 2A showed that MC from Anhui, Sichuan and Shandong declined ICAM-1 expression more obviously, while Hunan MC decreased TGF- $\beta 1$ expression more obviously than others (Figure 2B).

$\mathbf{A}$

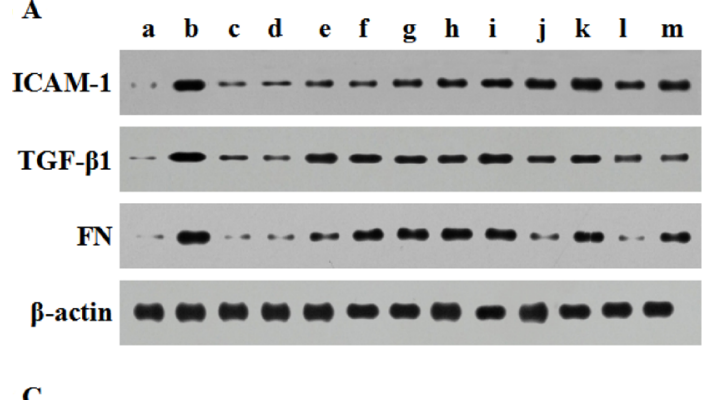

C

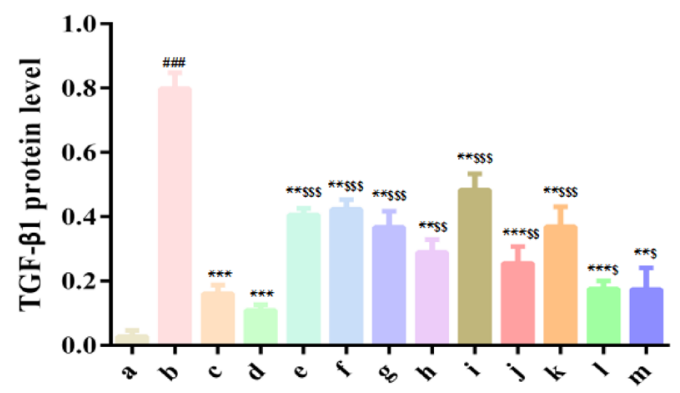

\section{Pharmacodynamic cluster analysis of MC from ten regions}

The classification results were given directly by cluster analysis. By the comparative analysis of physiological, biochemical indexes after administration of
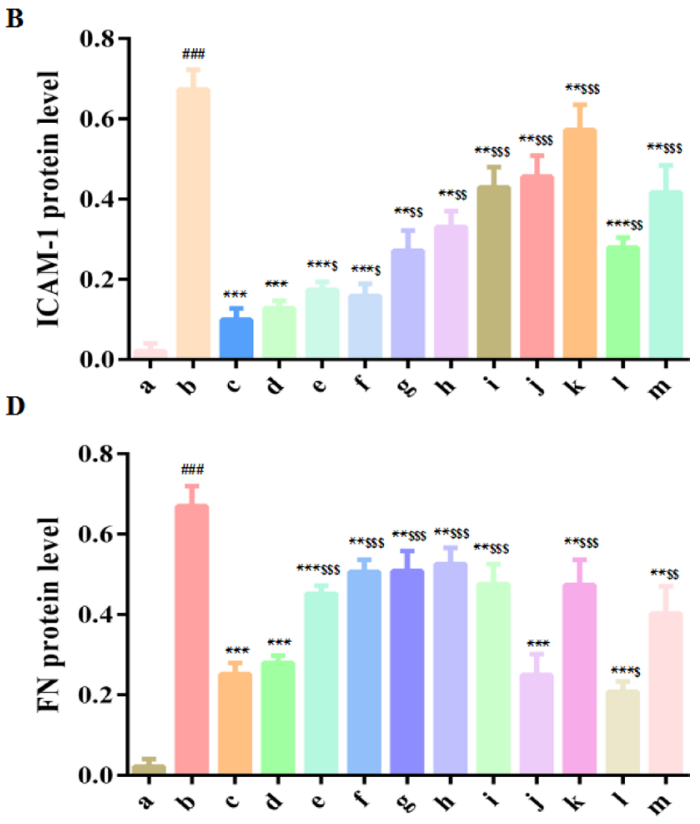

Figure 1: Comparison on the down-regulation of Moutan Cortex from different regions on protein expression levels of ICAM-1,TGF- $\beta 1$ and FN. HBZY-1 mesangial cells were treated with $200 \mu \mathrm{g} / \mathrm{mL}$ AGEs in the presence or absence of Moutan Cortex (MC) extract of $200 \mu \mathrm{g} / \mathrm{mL}$. Aminoguanidine of $10 \mu \mathrm{M}$ was used as the positive control while BSA (200 $\mu \mathrm{g} / \mathrm{mL}$ ) as blank control. (A) Western blotting was performed to compare the protein expression levels. (B-D) The grayscale scan results of ICAM-1, TGF- $\beta 1$ and FN. a, Control; b, $200 \mu \mathrm{g} / \mathrm{mL}$ AGEs; c, Positive control aminoguanidine group; "d-m" represent MC from Anhui, Guizhou, Zhejiang, Henan, Hunan, Hebei, Sichuan, Chongqing, Shandong, Gansu. Data are expressed as means $\pm \mathrm{SD}, n=3$. ${ }^{\# \#} P<0.001$ vs. BSA group; ${ }^{P}<0.05$, ${ }^{* *} P<0.01$ and ${ }^{* * *} P<0.001$ vs. AGEs group; ${ }^{{ }^{*}} P<0.05,{ }^{\text {ss }} P<0.01$ and ${ }^{\text {sss }} P<0.001$ vs. MC from Anhui group. 
Table 2: Effect of Moutan Cortex from different regions on CAT activity and GSH-Px activity $(n=6)$

\begin{tabular}{|c|c|c|c|}
\hline Groups & Dose $(\mathrm{g} / \mathrm{kg})$ & CAT (U/mL) & GSH-Px (U/L) \\
\hline Control & 0.1 & $5.42 \pm 0.41$ & $458.01 \pm 39.112$ \\
\hline Model & 0.1 & $2.54 \pm 0.15^{\# \#}$ & $319.83 \pm 18.62^{\# \#}$ \\
\hline Positive & 0.1 & $4.99 \pm 0.31^{*}$ & $442.63 \pm 16.60^{*}$ \\
\hline Gansu MC & 5 & $4.13 \pm 0.37^{* \$ \$ \$}$ & $367.05 \pm 26.09^{* S S \$}$ \\
\hline Chongqing MC & 5 & $4.47 \pm 0.28^{* \$ \$}$ & $415.45 \pm 25.19^{* \mathrm{~S}}$ \\
\hline Shandong MC & 5 & $3.51 \pm 0.15^{* * \$ \$ \$}$ & $450.34 \pm 57.28^{*}$ \\
\hline Sichuan MC & 5 & $5.22 \pm 0.14^{* \$}$ & $411.78 \pm 10.67^{* \$}$ \\
\hline Zhejiang MC & 5 & $3.62 \pm 0.07^{* * \$ \$ \$}$ & $419.48 \pm 12.24^{* \mathrm{~s}}$ \\
\hline Anhui MC & 5 & $5.50 \pm 0.14^{*}$ & $462.53 \pm 18.83^{* *}$ \\
\hline Hunan MC & 5 & $5.10 \pm 0.19^{* * \$}$ & $394.56 \pm 16.97^{* * s \$}$ \\
\hline Guizhou MC & 5 & $5.06 \pm 0.26^{* \$ \$}$ & $375.96 \pm 12.44^{* * \$ \$ \$ ~}$ \\
\hline Hebei MC & 5 & $3.45 \pm 0.26^{* * * \$ \$ \$}$ & $409.79 \pm 12.57^{* \mathrm{~S}}$ \\
\hline Henan MC & 5 & $3.48 \pm 0.11^{* * * \$ S S}$ & $424.55 \pm 39.95^{*}$ \\
\hline
\end{tabular}

Note: Control was control blank rats, model was DN model rats and positive was $0.1 \mathrm{~g} / \mathrm{kg} \mathrm{AG}$-treated rats. ${ }^{\#} P<0.01$ and ${ }^{\#} P<0.001$ vs. Control group; ${ }^{*} P<0.05,{ }^{* *} P<0.01$ and ${ }^{* * *} P<0.001$ vs. Model group $;{ }^{\$} P<0.05,{ }^{\$ \$} P<0.01$ and ${ }^{\$ \$ \$} P<0.001$ vs. Anhui MC group.

MC from 10 regions, which was proved that the efficacy was different between $\mathrm{MC}$ from different regions. Cluster analysis method could effectively classify different efficacy of MC. As shown in Figure 3A, MC from Guizhou, Zhejiang, Henan and Anhui were classified as a category due to the high efficacy. MC from Hunan, Hebei, Chongqing, Gansu, Sichuan and Shandong were classified as another class for the poor efficacy. It was indicated that there were no obvious differences of efficacy between $\mathrm{MC}$ from Anhui, Guizhou, Zhejiang and Henan. However, it also indicated the essential differences in quality of $\mathrm{MC}$ from different regions.

$\mathbf{A}$

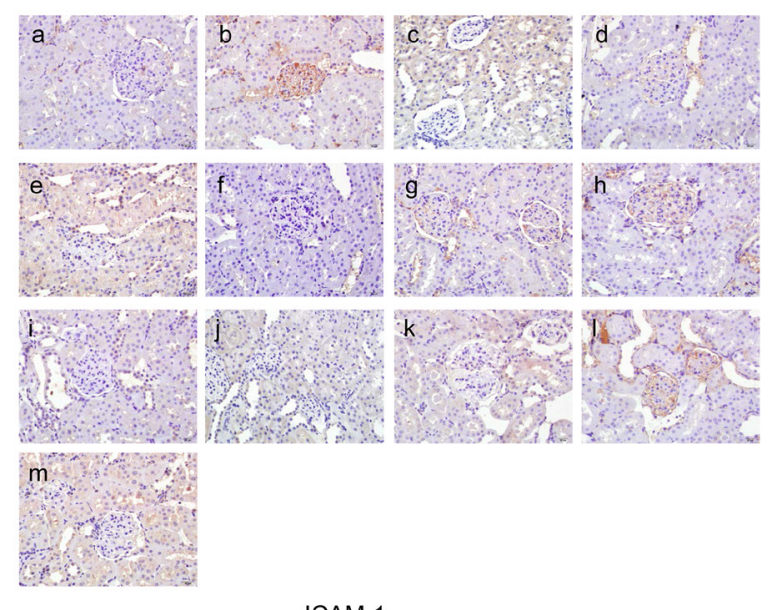

\section{MS analysis and comparison on the content of active components of $\mathrm{MC}$ from different regions}

MCs from 10 different regions were collected in our research (Figure 4A). The results in Table 3 showed the extraction rate of $\mathrm{MC}$ from different origins was different. As shown in Figure 4B and Table 4, it showed no significant difference in the categories of components but SCC with respect to the active components was different among $\mathrm{MC}$ from 10 regions. Thus, the cause of pharmacodynamic differences of MC from different regions was the stability of these efficacy components.

B

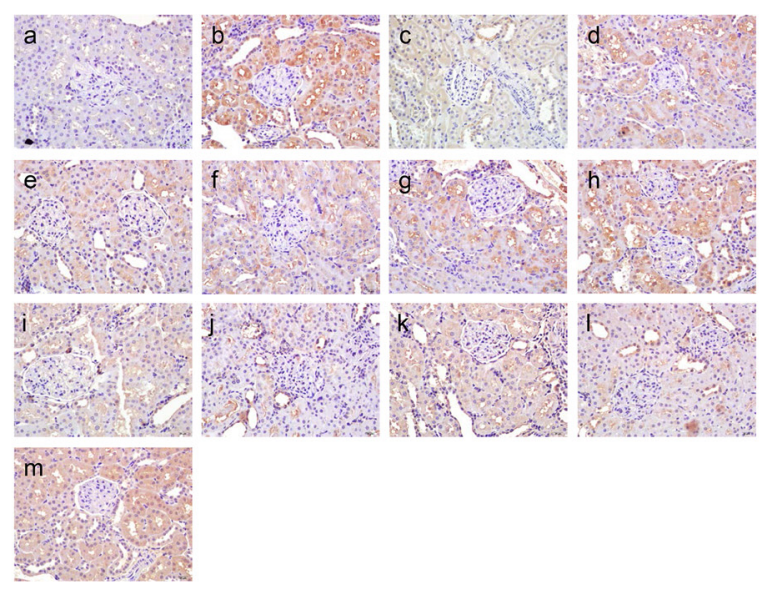

TGF- $\beta 1$

Figure 2: Effect of Moutan Cortex from different regions on ICAM-1 and TGF- $\beta 1$ protein expression levels in kidney of DN rats. After being treated with STZ and/or MC extract of $5 \mathrm{~g} / \mathrm{kg}$ or , immunohistochemistry was conducted to evaluate the expression levels of ICAM-1 (A) and TGF- $\beta 1$ (B) of renal tissues. "a" represents normal control; "b" represents model group (DN rats); "c" represents Positive control 0.1 g/kg AG; "d-m" represent Gansu, Chongqing, Shangdong, Sichuan, Zhejiang, Anhui, Hunan, Guizhou, Hebei, Henan. 
Table 3: Extract results of Moutan Cortex from different regions

\begin{tabular}{lccc}
\hline Province of origin & Herbs Weight $\mathbf{( k g )}$ & Extracts Weight $\mathbf{( g )}$ & Extraction rate (\%) \\
\hline Hunan & 1 & 182.38 & 18.24 \\
Anhui & 1 & 267.56 & 26.75 \\
Gansu & 1 & 156.72 & 15.67 \\
Chongqing & 1 & 231.74 & 23.17 \\
Sichuan & 1 & 100.99 & 10.10 \\
Guizhou & 1 & 215.50 & 21.55 \\
Henan & 1 & 227.55 & 22.76 \\
Hebei & 1 & 273.82 & 27.38 \\
Zhejiang & 1 & 194.32 & 19.43 \\
Shandong & 1 & 245.09 & 24.51 \\
\hline
\end{tabular}

A

Dendrogram using Average Linkage (Between Groups)

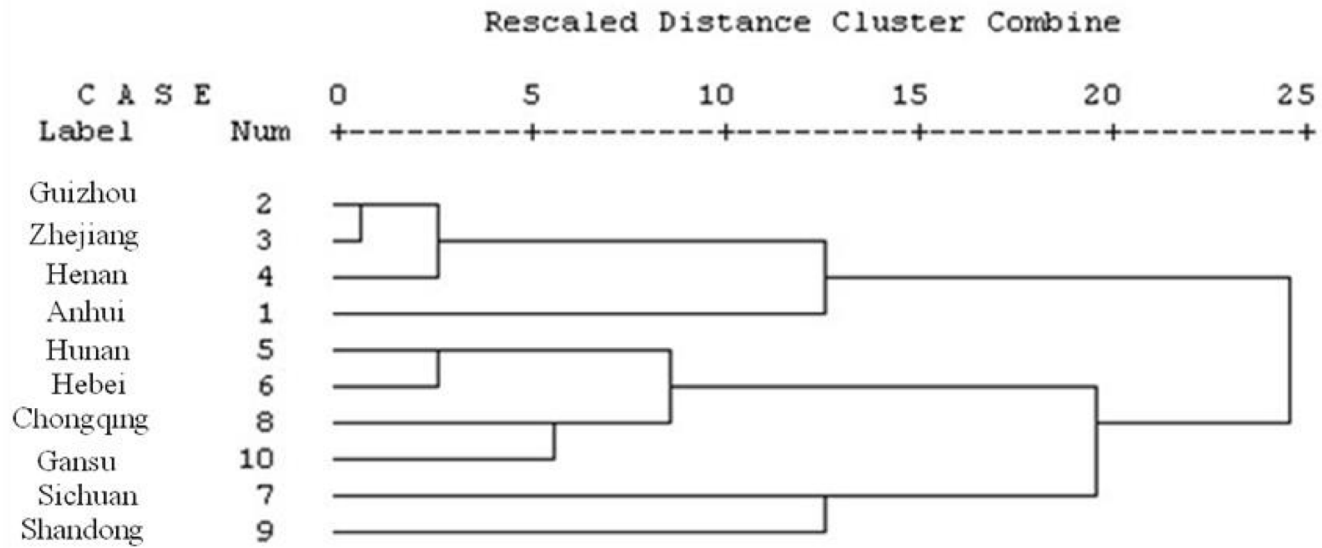

B

Dendrogram using dverage Linkage (Between Groups)

Rescaled Distance Cluster Combine

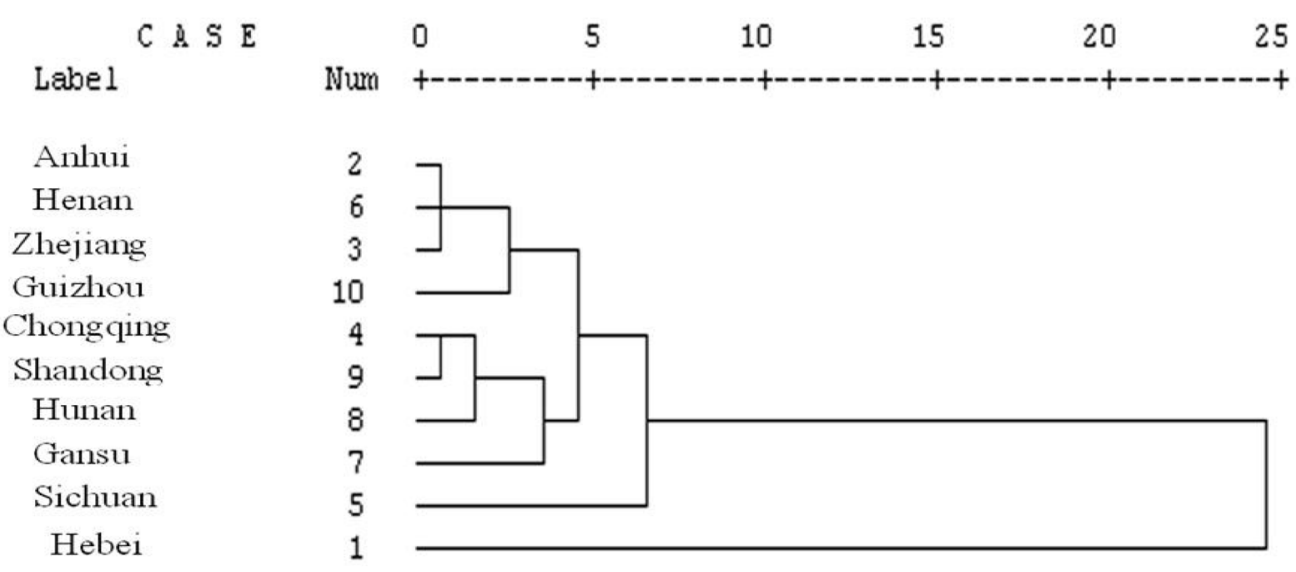

Figure 3: Cluster analysis of active ingredients (A) and efficacy dendrogram (B) of Moutan Cortex from different regions. SPSS16.0 sofeware was performed for Cluster analysis. 
The active components in each MC were identified by LC-MS analysis. Peaks (No. 2, 7, 13, 14, 16, 17, 18, 19, 20, 21, 22, 23 and 24) in Table 4 were identified as mudanoside B, paeoniflorin sulfonate, oxypaeoniflorin, paeoniflorin, trigalloyl glucose, tetragalloyl glucose, tetragalloyl glucose, pentagalloyl glucose, hexagalloyl glucose, galloylpaeoniflorin, benzoylpaeoniflorin, mudanoside A, paeonol, as we published before (Figure 4C) [9]. The component analysis for $\mathrm{MC}$ from different regions showed in Table 5 revealed that $\mathrm{MC}$ from each region had its own unique intrinsic ratio of active components.

\section{Cluster analysis for MC from different regions}

The peak areas of 13 chemical compositions in MC from different regions were set as quantitative traits to conduct cluster analysis by SPSS16.0. Combined with the cluster analysis of peak area, MC from Zhejiang, Henan and Anhui were considered as a class for the same constituted composition. It was indicated that geographical advantages would affect the quality of the CMM. MC from Zhejiang, Henan and Anhui were grouped as another class due to the similar constitution. As shown in Figure 3B, Hebei MC was divided into a separate class due to the significant difference in the ratio of active components compared with Anhui MC.

\section{Principal component analysis for active components in MC}

A few indicators could be filtered by principal component analysis to reflect the overall message. To select representative compounds among 13, the peak areas of 13 compounds (mudanoside B, paeoniflorin sulfonate, oxypaeoniflorin, paeoniflorin, trigalloyl glucose, tetragalloyl glucose, tetragalloyl glucose, pentagalloyl glucose, hexagalloyl glucose, galloylpaeoniflorin, benzoylpaeoniflorin, mudanoside A, paeonol) from 10 regions were analyzed by principal component analysis using SPSS 16.0. The 13 compounds were set to $\mathrm{x} 1 \sim \mathrm{x} 13$ as variables to establish the data file of 10 regions. As shown in Table 6 and Table 7, they revealed that the cumulative contribution rate of the first six principal components main up to $95.08 \%$, which indicated that the six representative compounds could be used as the main components due to their high content. The six main components were known as oxypaeoniflorin, paeoniflorin, benzoylpaeoniflorin, paeonol, trigalloyl glucose and pentagalloyl glucose by correlation matrix analysis. These six components were preferred as the representative active components contributing to the efficacy.

\section{Effects of active components on SOD activity and MDA content in AGEs-induced HBZY-1 mesangial cells}

We measured SOD activity and MDA content to evaluate the activity of these components. As shown in Figures 5A and 6B, SOD activity was significantly decreased whereas MDA content was increased remarkably by AGEs, compared with the control group $(P<0.05)$. After the treatment of these active components, SOD activity was increased while MDA content was decreased, which means that oxypaeoniflorin, paeoniflorin, benzoylpaeoniflorin, paeonol, trigalloyl glucose and pentagalloyl glucose can reduce the AGEsinduced oxidative stress.

\section{Effects of active components on IL-6 and MCP-1 levels in AGEs-induced HBZY-1 mesangial cells}

The contents of IL-6 and MCP-1 in supernatants of HBZY-1 mesangial cells were measured by ELISA kits according to the manufacturer's protocols. As shown in Figures $5 \mathrm{C}$ and $6 \mathrm{D}$, the contents of IL-6 and MCP -1 could be increased by AGEs significantly $(P<0.001)$. After the treatment of these active components, the contents of IL-6 and MCP-1 were reduced significantly $(P<0.05, P$ $<0.01, P<0.001)$. The results showed that the screened active components (oxypaeoniflorin, paeoniflorin, benzoylpaeoniflorin, paeonol, trigalloyl glucose and pentagalloyl glucose) could reduce IL-6 and MCP-1 contents in AGEs-induced mesangial cells.

\section{Reduction of active components on $\mathrm{FN}$ expression in AGEs-induced HBZY-1 cells}

As shown in Figure 6, FN protein expression was reduced by the active components $(P<0.01, P<0.001)$ when compared with the AGEs-induced cells. Statistical analysis was performed based on the value of scanned grayscale. Results showed that these six components could inhibit FN secreting.

To clarify the pharmacological effects of oxypaeoniflorin, paeoniflorin, trigalloyl glucose, pentagalloyl glucose, benzoylpaeoniflorin and paeonol, their effects on pathological phenomena of AGEs-induced HBZY1 mesangial cells, such as oxidative stress, inflammation, thickening of the basement membrane were observed and detected. The results showed that the six preferred active components could increase SOD activity, decrease MDA content, reduce the content of IL-6, MCP-1 and FN protein.

\section{SCC in Anhui geoherb MC and Heibei non- geoherb MC}

As shown in Figure 3B, the result of cluster analysis showed that MC from Zhejiang, Henan, Anhui were considered as a class for the same constituted compositions, while Hebei MC was divided into a separate class for the significant difference in active components compared with the Anhui MC. Contents of six active components of geoherb (Anhui) and non-geoherb (Hebei) MC were measured. The test solutions of Anhui MC and Hebei MC 
Table 4: Peak area comparison of 20 compounds in Moutan Cortex from different regions

\begin{tabular}{ccccccccccc}
\hline Peak No. & Hunan & Anhui & Gansu & $\begin{array}{c}\text { Chong } \\
\text { qing }\end{array}$ & Sichuan & Guizhou & Henan & Hebei & $\begin{array}{c}\text { Zhe } \\
\text { jiang }\end{array}$ & $\begin{array}{c}\text { Shan } \\
\text { dong }\end{array}$ \\
\hline 1 & 7342.3 & 8021.7 & 4402.1 & 2171.7 & 9639.1 & 5049.3 & 3887.5 & 7240.2 & 2832.0 & 6493.9 \\
2 & 1983.0 & 1744.7 & 1764.54 & 788.34 & 908.34 & 1039.2 & 845.2 & 775.5 & 615.8 & 1033.6 \\
3 & 4449.1 & 4481.5 & 5998.1 & 8022.4 & 8678.6 & 4528.1 & 3659.3 & 2519.1 & 5913.4 & 5375.7 \\
4 & 209.0 & 442.3 & 489.3 & 484.0 & 775.0 & 449.1 & 375.8 & 294.3 & 477.4 & 512.1 \\
5 & 8474.4 & 10000.1 & 10784.5 & 5382.3 & 5070.0 & 5482.3 & 10374.7 & 2541.2 & 3176.4 & 12211.6 \\
6 & 3618.3 & 3088.4 & 2090.1 & 1880.3 & 3367.6 & 1774.6 & 1759.0 & 2876.6 & 1561.5 & 2999.7 \\
7 & 298.83 & 440.9 & 436.34 & 634.54 & 245.45 & 276.63 & 213.3 & 397.16 & 155.4 & 653.3 \\
8 & 1530.7 & 1635.7 & 2202.6 & 2337.6 & 3287.5 & 2173.7 & 1310.4 & 837.8 & 2084.6 & 1706.6 \\
9 & 2978.1 & 2973.0 & 2155.1 & 3334.8 & 2452.0 & 1375.9 & 2244.6 & 651.0 & 830.3 & 2323.0 \\
10 & 1242.1 & 1195.4 & 1101.9 & 1040 & 2975.2 & 1018.9 & 938.5 & 1182.2 & 2298.5 & 1465.5 \\
11 & 764.1 & 611.2 & 600.4 & 490.5 & 1070.3 & 591.2 & 472.6 & 226.2 & 992.9 & 866.9 \\
12 & 4173.1 & 3413.5 & 1363.4 & 702.4 & 2536.9 & 2004.2 & 2143.0 & 1067.8 & 1634.1 & 3729.5 \\
13 & 3186.3 & 2454.0 & 8364.3 & 7213.0 & 14956.8 & 3729.9 & 1272.5 & 11017.5 & 825.1 & 3653.5 \\
14 & 23868.3 & 18626.6 & 19801.2 & 12850.1 & 26839.0 & 16328.4 & 9691.1 & 12806.7 & 6466.7 & 27246.2 \\
15 & 927.2 & 683.6 & 921.8 & 1375.0 & 1356.9 & 765.9 & 752.3 & 488.9 & 1969.5 & 1138.1 \\
16 & 673.23 & 958.6 & 743.35 & 1106.34 & 973.11 & 605.82 & 463.7 & 345.4 & 339.1 & 452.4 \\
17 & 975.34 & 1159.9 & 744.0 & 1254.34 & 1054.34 & 739.63 & 561.1 & 662.3 & 441.0 & 645.2 \\
18 & 2069.3 & 1198.4 & 1316.0 & 151.2 & 485.4 & 718.98 & 536.7 & 1759.7 & 426.6 & 3891.8 \\
19 & 4971.1 & 4822.9 & 1689.2 & 981.7 & 1966.0 & 3031.4 & 2906.9 & 1936.9 & 2165.7 & 3966.8 \\
20 & 1507.5 & 1033.2 & 1368.7 & 1214.7 & 2390.3 & 691.4 & 517.7 & 382.7 & 413.5 & 1673.0 \\
21 & 2185.4 & 1335.4 & 1900.2 & 1752.9 & 2139.6 & 864.7 & 772.4 & 1376.6 & 489.7 & 2029.9 \\
22 & 283.8 & 1890.0 & 282.3 & 860.0 & 356.6 & 1116.7 & 1038.2 & 1074 & 618.8 & 306.2 \\
23 & 898.0 & 1610.8 & 1062.6 & 1733.5 & 1841.6 & 1057.7 & 730.0 & 913.1 & 539.4 & 1062.6 \\
24 & 51959.9 & 26336.4 & 25964.1 & 20926.5 & 27467.6 & 16480.1 & 13617.8 & 14555.7 & 9426.5 & 29885.6 \\
\hline
\end{tabular}

were a mixture of oxypaeoniflorin, paeoniflorin, trigalloyl glucose, pentagalloyl glucose, benzoylpaeoniflorin and paeonol in the ratio 1:7.5:0.3:0.4:0.7:10.7, respectively. The concentrations of each component were oxypaeoniflorin $(1.94 \mu \mathrm{g} / \mathrm{mL})$, paeoniflorin $(14.56 \mu \mathrm{g} / \mathrm{mL})$, trigalloyl glucose $(0.58 \mu \mathrm{g} / \mathrm{mL})$, pentagalloyl glucose $(0.58 \mu \mathrm{g} /$ $\mathrm{mL})$, benzoylpaeoniflorin $(2.04 \mu \mathrm{g} / \mathrm{mL})$ and paeonol $(20.78 \mu \mathrm{g} / \mathrm{mL})$ in cell experiment. Ratio of the six active components in geoherb MC was 1:7.5:0.3:0.4:0.7:10.7, the result was consistent with ratio of peak area in Table 5. As shown in Table 8, the ratio of Hebei non-geoherb MC was 1:1.2:0.03:0.03:0.1:1.3, similar with the result of comparison of the peak area. The results demonstrated that the prevention and treatment of geoherb $\mathrm{MC}$ on DN was due to SCC instead of the categories of compounds.

\section{Effects of SCC transformation of non-geoherb MC on SOD activity, and MDA, IL-6, MCP-1 levels}

The results of Table 9 demonstrated that, after recombination, the efficacy was superior to the geoherb one. The SCC of oxypaeoniflorin, paeoniflorin, trigalloyl glucose, pentagalloyl glucose, benzoylpaeoniflorin and paeonol in Hehei was adjusted from 1:1.2:0.03:0.03:0.1:1.3 to $1: 7.5: 0.3: 0.4: 0.7: 10.7$ as Anhui MC's. SOD activity of Anhui was $7.71 \pm 0.98 \mathrm{U} / \mathrm{mL}$, while Hebei recombination one was $7.17 \pm 0.53 \mathrm{U} / \mathrm{mL}$. MDA content of Anhui was $0.46 \pm 0.05 \mathrm{nM}$ while Hebei recombination one was $0.43 \pm 0.06 \mathrm{nM}$. Meanwhile, IL-6 and MCP-1 contents of Anhui MC were $3.20 \pm 0.1 \mathrm{ng} / \mathrm{L}$ and $13.67 \pm 0.12 \mathrm{ng} / \mathrm{L}$, respectively. These two contents in Hebei recombination were $3.62 \pm 0.10 \mathrm{ng} / \mathrm{L}$ and $3.85 \pm$ $0.16 \mathrm{ng} / \mathrm{L}$. There was no significant difference in efficacy between Hebei recombination and Anhui MC. This further validated that $\mathrm{SCC}$ acted as a crucial contributor to the quality consistency of $\mathrm{MC}$ which was related to the better efficacy.

\section{DISCUSSION}

CMM is the material basis of TCM to prevent or treat disease. Its quality directly affects the clinical efficacy. Geoherbs are the representative with particular 
A

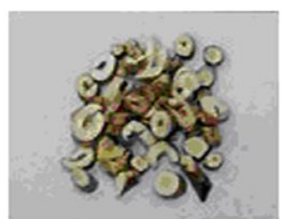

Gansu

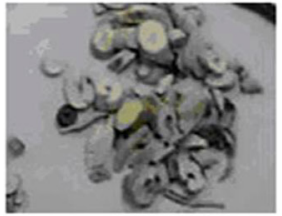

Guizhou

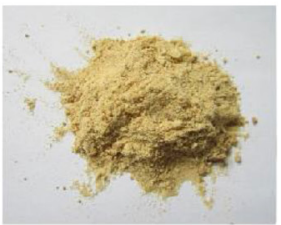

Gansu

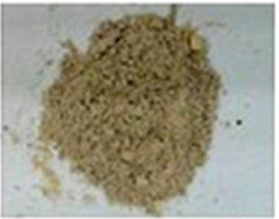

Guizhou

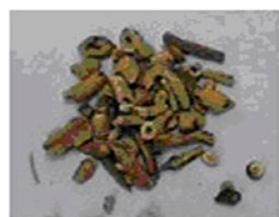

Hunan

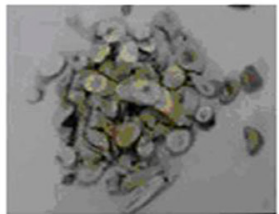

Hebei

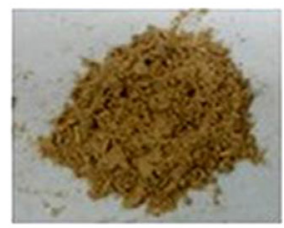

Hunan

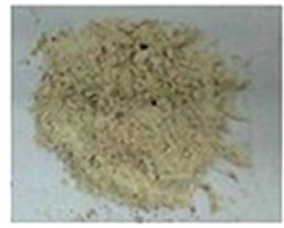

Hebei

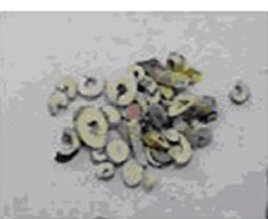

Shandong

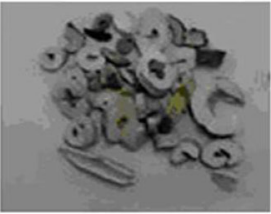

Henan

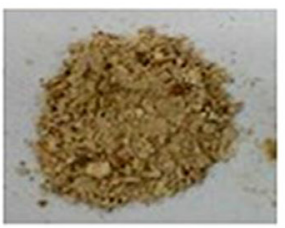

Shandong

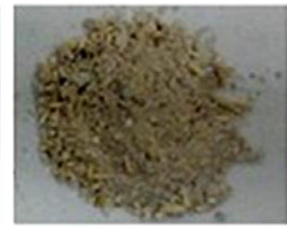

Henan

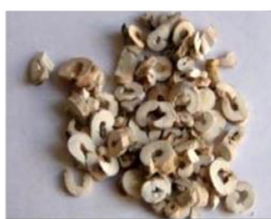

Zhejiang

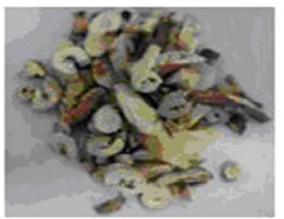

Chongqing

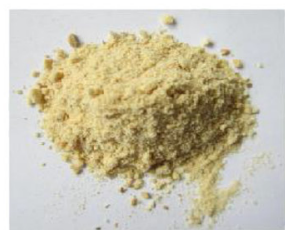

Zhejiang

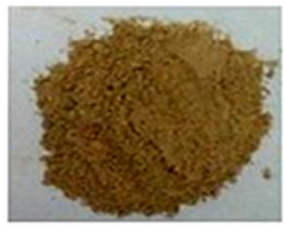

Chongqing
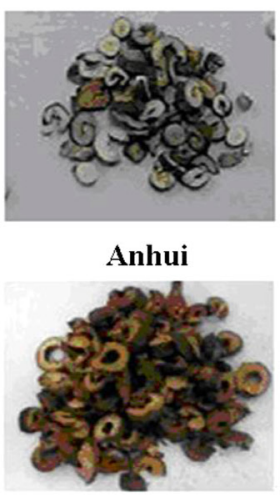

Sichuan

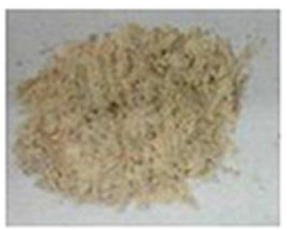

Anhui

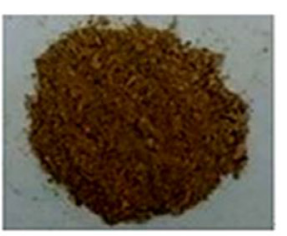

Sichuan

B

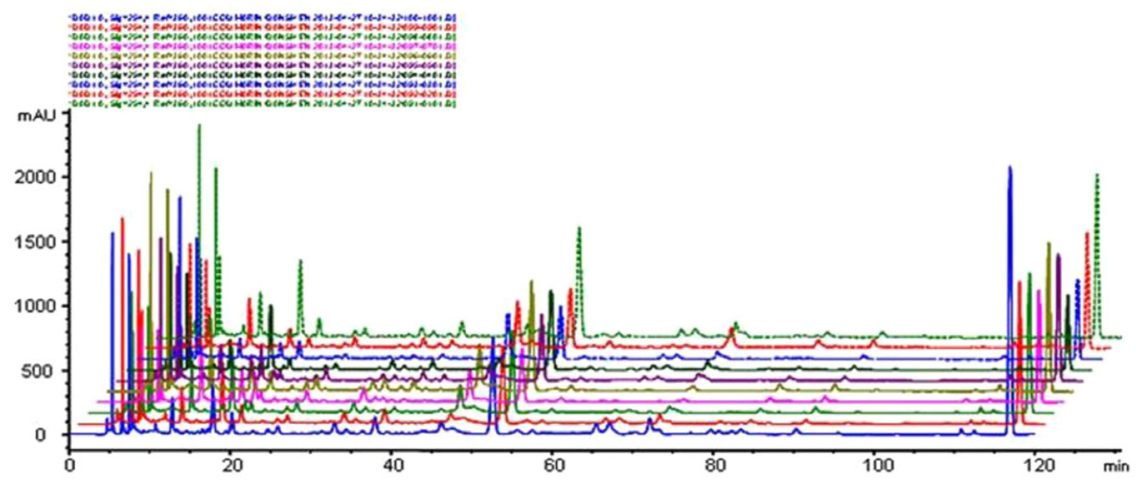

C

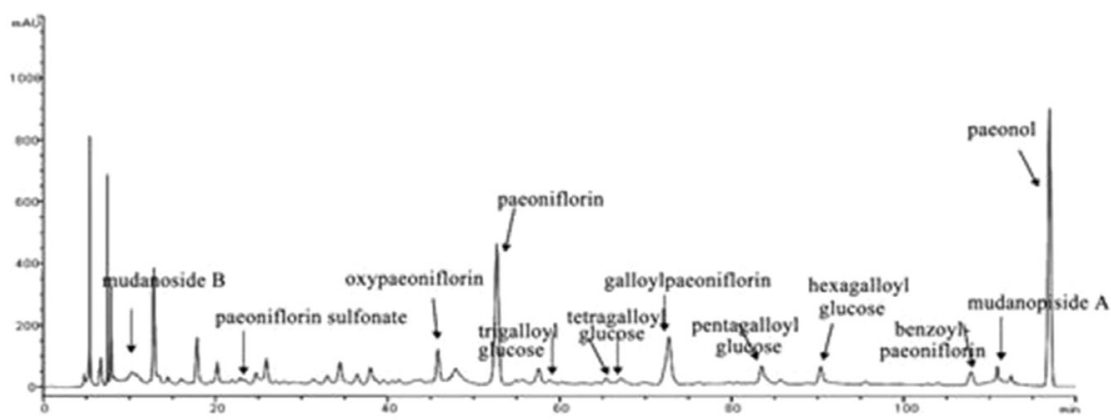

Figure 4: Pieces characteristics, powder characteristics and composition differences analysis. (A) Characteristics comparison of Moutan Cortex and their powder from different regions. (B) HPLC chromatogram of Moutan Cortex from different regions. The order of absorption peaks that from front to back was from A to J. (C) The chromatogram of active ingredients in Moutan Cortex. 
Table 5: SCC in Moutan Cortex from different regions

\begin{tabular}{ll}
\hline Province of origins & Structural composition of components \\
\hline Hunan & $1: 0.1: 1.1: 12.0: 0.3: 0.4: 1.0: 2.5: 0.7: 1.1: 0.1: 0.4: 26.2$ \\
Anhui & $1: 0.2: 1.4: 10.4: 0.5: 0.6: 0.6: 2.7: 1.1: 0.7: 1.0: 1.1: 14.9$ \\
Gansu & $1: 0.2: 4.7: 11.2: 0.4: 0.4: 0.7: 0.9: 0.7: 1.0: 0.1: 0.6: 14.7$ \\
Chongqing & $1: 0.8: 9.1: 16.3: 1.4: 1.5: 0.1: 1.2: 1.5: 2.2: 1.0: 2.1: 26.5$ \\
Sichuan & $1: 0.2: 16.4: 29.5: 1.0: 1.1: 0.5: 2.1: 2.6: 2.3: 0.3: 2.0: 30.2$ \\
Guizhou & $1: 0.2: 3.5: 15.7: 0.5: 0.7: 0.6: 2.9: 0.6: 0.8: 1.1: 1.0: 15.8$ \\
Henan & $1: 0.2: 1.5: 13.9: 0.5: 0.6: 0.6: 3.4: 0.6: 0.9: 1.2: 0.8: 16.1$ \\
Hebei & $1: 0.5: 14.2: 16.5: 0.4: 0.8: 2.2: 2.4: 0.4: 1.7: 1.3: 1.1: 18.7$ \\
Zhejiang & $1: 0.2: 1.3: 10.5: 0.5: 0.7: 0.6: 3.5: 0.6: 0.7: 1.0: 0.8: 15.3$ \\
Shandong & $1: 0.6: 3.5: 26.3: 0.4: 0.6: 3.7: 3.8: 1.6: 1.9: 0.2: 1.0: 28.9$ \\
\hline
\end{tabular}

A

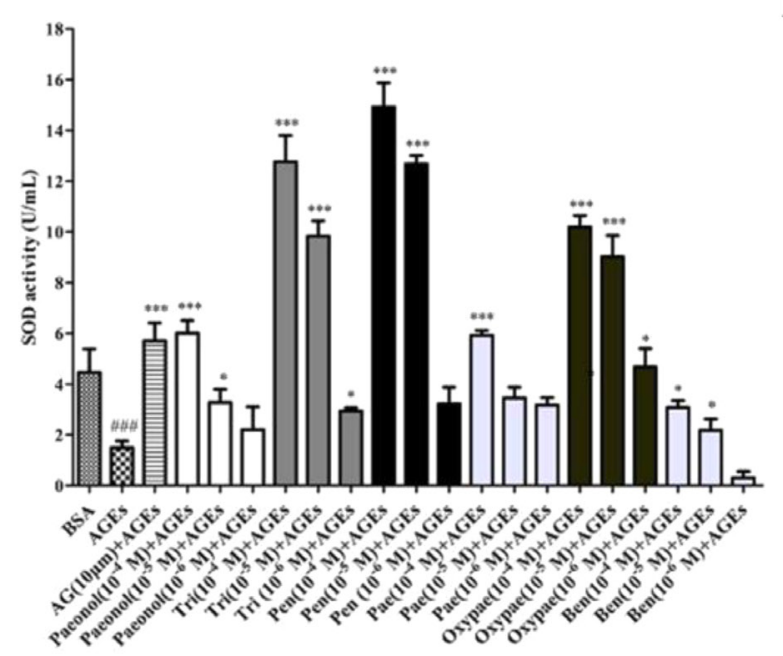

C

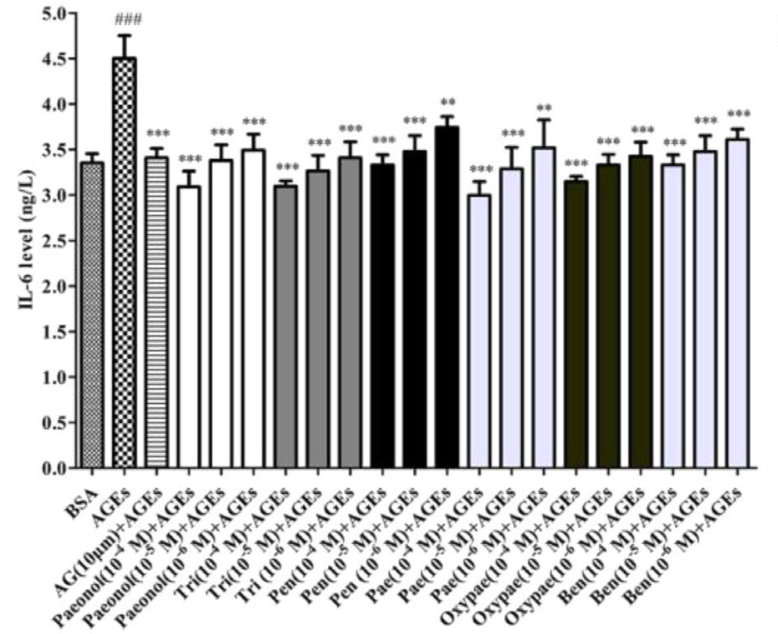

B

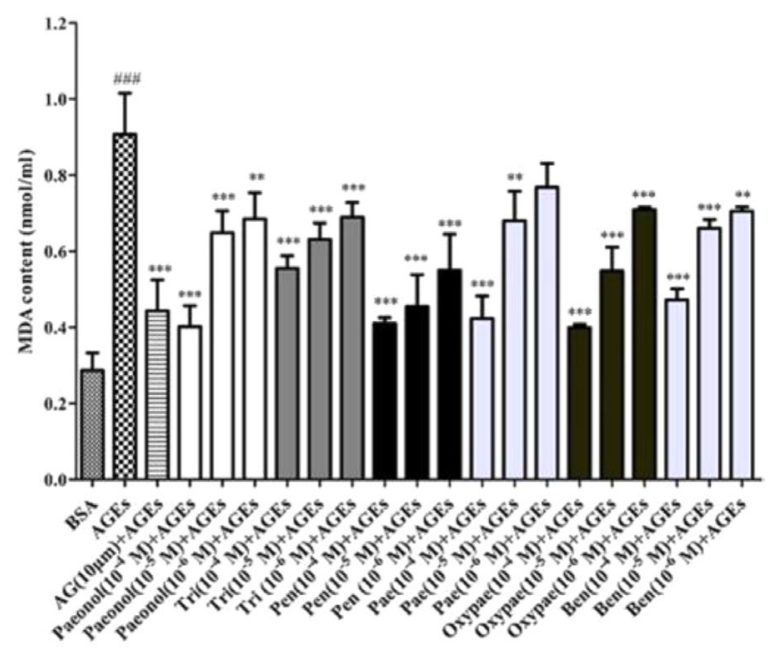

D

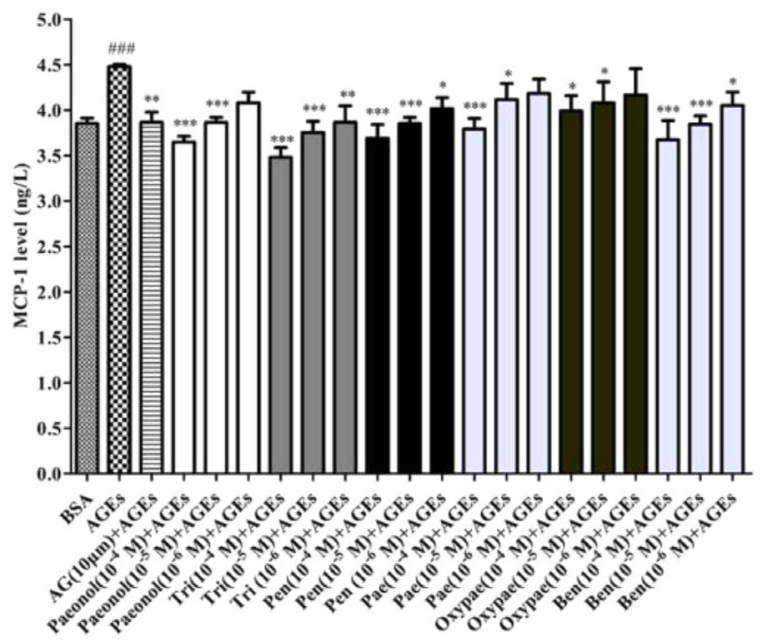

Figure 5: Effect of components on AGEs-induced SOD activity (A), MDA (B), IL-6 (C) and MCP-1 (D) content. The determination of these detection indicators according to manufacturer's instructions. OD value of samples was determined at $550 \mathrm{~nm}$ for SOD, $532 \mathrm{~nm}$ for MDA, $450 \mathrm{~nm}$ for IL-6 and MCP-1. Data are expressed as means \pm SD, $n=3 .{ }^{\# \#} P<0.001$ vs. BSA group; ${ }^{*} P<0.05,{ }^{* * *} P<0.01$ and ${ }^{* * *} P<$ 0.001 vs. AGEs group. 
Table 6: Characteristic value and the variance contribution rate

\begin{tabular}{|c|c|c|c|c|c|c|}
\hline \multirow{2}{*}{ Compents } & \multicolumn{3}{|c|}{ Initial eigenvalues } & \multicolumn{3}{|c|}{ Load of square extracting } \\
\hline & Eigenvalues & Variance $\%$ & Cumulative \% & Total & Variance $\%$ & Cumulative $\%$ \\
\hline Mudanoside B & 5.645 & 43.421 & 43.421 & 5.645 & 43.421 & 43.421 \\
\hline Paeoniflorin sulfonate & 2.981 & 22.932 & 66.353 & 2.981 & 22.932 & 66.353 \\
\hline Oxypaeoniflorin & 1.993 & 15.331 & 81.684 & 1.993 & 15.331 & 81.684 \\
\hline Paeoniflorin & 1.165 & 8.959 & 90.643 & 1.165 & 8.959 & 90.643 \\
\hline Trigalloyl glucose & 0.570 & 4.385 & 95.082 & 0.570 & 4.385 & 95.082 \\
\hline Tetragalloyl glucose & 0.422 & 3.250 & 98.278 & 0.422 & 3.250 & 98.278 \\
\hline Tetragalloyl glucose & 0.197 & 1.513 & 99.791 & & & \\
\hline Pentagalloyl glucose & 0.021 & 0.159 & 99.950 & & & \\
\hline Hexagalloyl glucose & 0.007 & 0.050 & 100.000 & & & \\
\hline Galloylpaeoniflorin & $6.82 \mathrm{E}-16$ & $5.24 \mathrm{E}-15$ & 100.000 & & & \\
\hline Benzoylpaeoniflorin & $4.09 \mathrm{E}-16$ & $3.15 \mathrm{E}-15$ & 100.000 & & & \\
\hline Mudanoside A & $8.61 \mathrm{E}-17$ & $6.62 \mathrm{E}-16$ & 100.000 & & & \\
\hline Paeonol & $-2.8 \mathrm{E}-16$ & $-2.15 \mathrm{E}-15$ & 100.000 & & & \\
\hline
\end{tabular}

Table 7: Matrix of relative principal components

\begin{tabular}{lcccccc}
\hline \multirow{2}{*}{ Compents } & \multicolumn{7}{c}{ principal components } \\
\cline { 2 - 6 } & $\mathbf{1}$ & $\mathbf{2}$ & $\mathbf{3}$ & $\mathbf{4}$ & $\mathbf{5}$ & $\mathbf{6}$ \\
\hline Mudanoside B & 0.560 & 0.432 & 0.461 & -0.258 & 0.452 & 0.560 \\
Paeoniflorin sulfonate & 0.502 & -0.038 & 0.003 & 0.531 & 0.045 & 0.462 \\
Oxypaeoniflorin & 0.460 & -0.508 & 0.775 & -0.061 & -0.123 & 0.802 \\
Paeoniflorin & 0.878 & 0.772 & -0.152 & -0.025 & 0.672 & -0.052 \\
Trigalloyl glucose & 0.685 & -0.606 & 0.334 & -0.092 & 0.245 & -0.035 \\
Tetragalloyl glucose & 0.734 & -0.484 & 0.427 & 0.007 & 0.102 & 0.568 \\
Tetragalloyl glucose & 0.344 & 0.304 & -0.179 & 0.478 & 0.357 & 0.268 \\
Pentagalloyl glucose & 0.238 & 0.709 & 0.588 & -0.061 & -0.017 & 0.433 \\
Hexagalloyl glucose & 0.893 & -0.039 & -0.270 & -0.180 & -0.082 & 0.168 \\
Galloylpaeoniflorin & 0.794 & -0.102 & -0.255 & 0.046 & 0.662 & -0.485 \\
Benzoylpaeoniflorin & -0.319 & -0.308 & 0.545 & 0622 & -0.381 & 0.544 \\
Mudanoside A & 0.705 & -0.643 & 0.172 & 0.117 & 0.161 & -0.066 \\
Paeonol & 0.941 & 0.470 & 0.141 & -0.241 & -0.007 & 0.938 \\
\hline
\end{tabular}

area, excellent quality, and good efficacy making them become the proverbial superiors of TCM [1]. The material basis of geoherbs efficacy is their internal components. Its SCC contributes to the level of efficacy and the pros and cons of quality [12]. The scientific connotation of geoherbs quality and the relationship between the quality and efficacy of geoherbs are the emphases and difficulty in the study of geoherbs. $\mathrm{MC}$ is one of the commonly used TCM with significant activity against DN [13]. The prevention of MC on DN included series pharmacological effects, including its improvement on the basement membrane thickening, oxidative stress, inflammation, and so on. In our present study, body weight, the content of blood glucose, urinary albumin and serum creatinine of DN rats were determined to reflect the treatment of MC from 10 regions. Among them, Anhui MC hold more significant reduction on the blood glucose, $24 \mathrm{~h}$ urine protein and serum creatinine. Therefore, Anhui MC, geoherb one, had a better anti-DN efficacy than other nongeoherb MCs (Figure 2). To further confirm the superior effect of Anhui MC on the treatment of DN, CAT, GSHPx activity, the protein expression of ICAM- 1 , TGF- $\beta 1$ and FN were detected to reflect the efficacy of MC from different regions. Finally, these samples were classified by cluster analysis, results indicated that geoherb MC from Anhui Tongling held the best efficacy in the treatment 
of DN. MC from Guizhou, Zhejiang and Henan were classified as a category due to their similar efficacy. MC from Hunan, Hebei, Chongqing, Gansu, Sichuan and Shandong were classified as another classification based on their poor efficacy .

The material basis of geoherbs is composed of various components with a stable compositional characteristic, related to the stable SCC but not new compounds. Structural inconsistencies directly result in pharmacodynamic differences. Based on the overall concept of Chinese medicine and "component structure theory" [3], we compared the difference of efficacy and components between geoherb and non-geoherb MC. The efficacy of $\mathrm{MC}$ from different regions was not same.
Unique stable SCC in geoherb MC contributed to a better efficacy on prevention and treatment of DN. Our study confirmed the different traits and extraction rate of $\mathrm{MC}$ from different regions, but there was no significant difference in the types of active components. The cluster analysis of efficacy component was introduced combined with peak areas of MC from 10 regions. After analyzing the belonging of the 13 components, we found that composition of Zhejiang was the same as Henan and Anhui MC. The composition of Hebei MC was quite different with Anhui. According to PCA, SCC of six components (oxypaeoniflorin, paeoniflorin, trigalloyl glucose, pentagalloyl glucose, benzoylpaeoniflorin and paeonol) have been recombinated for further study. These
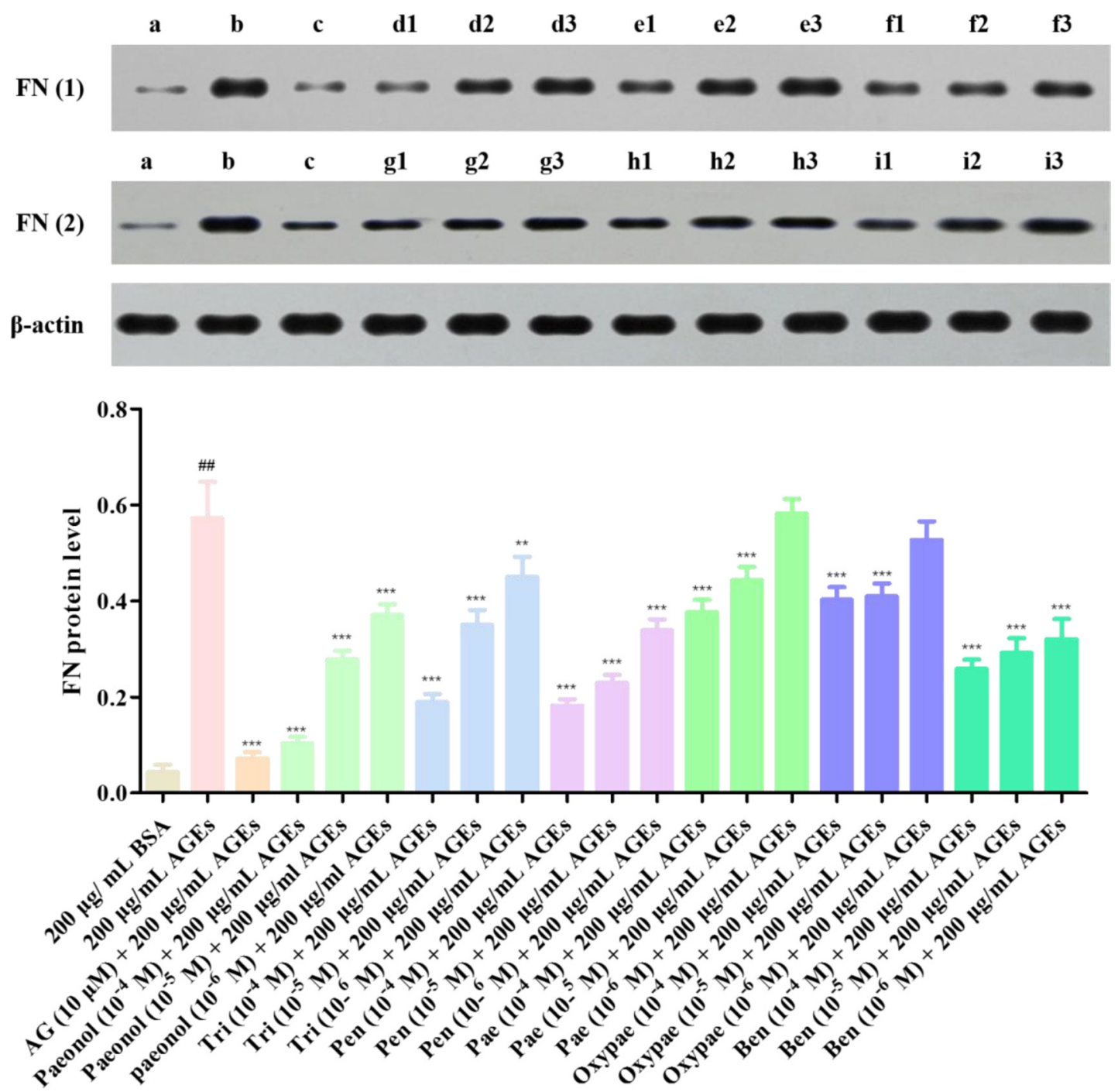

Figure 6: Effect of components on FN protein expression in AGEs-induced mesangial cells. a, $200 \mu \mathrm{g} / \mathrm{mL} \mathrm{BSA}$; b, 200 $\mu \mathrm{g} / \mathrm{mL}$ AGEs; c, AG $(10 \mu \mathrm{M})+200 \mu \mathrm{g} / \mathrm{mL}$ AGEs; d1, paeonol $\left(10^{-4} \mathrm{M}\right)+200 \mu \mathrm{g} / \mathrm{mL}$ AGEs; d2, paeonol $\left(10^{-5} \mathrm{M}\right)+200 \mu \mathrm{g} / \mathrm{ml} \mathrm{AGEs} ; \mathrm{d} 3$, paeonol $\left(10^{-6} \mathrm{M}\right)+200 \mu \mathrm{g} / \mathrm{mL}$ AGEs; e1, Tri $\left(10^{-4} \mathrm{M}\right)+200 \mu \mathrm{g} / \mathrm{mL}$ AGEs; e2, Tri $\left(10^{-5} \mathrm{M}\right)+200 \mu \mathrm{g} / \mathrm{mL}$ AGEs; e3, Tri $\left(10^{-6} \mathrm{M}\right)+200 \mu \mathrm{g} /$ mL AGEs; f1, Pen $\left(10^{-4} \mathrm{M}\right)+200 \mu \mathrm{g} / \mathrm{mL}$ AGEs; f2, Pen $\left(10^{-5} \mathrm{M}\right)+200 \mu \mathrm{g} / \mathrm{mL}$ AGEs; f3, Pen $\left(10^{-6} \mathrm{M}\right)+200 \mu \mathrm{g} / \mathrm{mL}$ AGEs; g1, Pae $\left(10^{-4} \mathrm{M}\right)$ $+200 \mu \mathrm{g} / \mathrm{mL}$ AGEs; g2, Pae $\left(10^{-5} \mathrm{M}\right)+200 \mu \mathrm{g} / \mathrm{ml} \mathrm{AGEs;} \mathrm{g3,} \mathrm{Pae}\left(10^{-6} \mathrm{M}\right)+200 \mu \mathrm{g} / \mathrm{mL}$ AGEs; h1, Oxypae $\left(10^{-4} \mathrm{M}\right)+200 \mu \mathrm{g} / \mathrm{mL}$ AGEs; h2, Oxypae $\left(10^{-5} \mathrm{M}\right)+200 \mu \mathrm{g} / \mathrm{mL}$ AGEs; h3, Oxypae $\left(10^{-6} \mathrm{M}\right)+200 \mu \mathrm{g} / \mathrm{mL}$ AGEs; i1, Ben $\left(10^{-4} \mathrm{M}\right)+200 \mu \mathrm{g} / \mathrm{mL}$ AGEs; i2, Ben $\left(10^{-5} \mathrm{M}\right)+$ $200 \mu \mathrm{g} / \mathrm{mL}$ AGEs; i3, Ben $\left(10^{-6} \mathrm{M}\right)+200 \mu \mathrm{g} / \mathrm{mL}$ AGEs. Data are expressed as means $\pm \mathrm{SD}, n=6 .{ }^{\# \#} P<0.001 \mathrm{vs}$. BSA group; ${ }^{*} P<0.05$, ${ }^{* *} P<0.01$ and ${ }^{* * *} P<0.001$ vs. AGEs group.. 
Table 8: Content of six components in geoherb (Anhui) and non-geoherb (Hebei) Moutan Cortex and their compositions structure $(\mu \mathrm{g} / \mathrm{mL})$

\begin{tabular}{cccccccc}
\hline Origins & Oxypaeoniflorin & Paeoniflorin & $\begin{array}{c}\text { Trigalloyl } \\
\text { glucose }\end{array}$ & $\begin{array}{c}\text { Pentagalloyl } \\
\text { glucose }\end{array}$ & Benzoylpaeoniflorin & Paeonol & Ratio \\
\hline Anhui & 12.98 & 97.47 & 5.06 & 5.45 & 9.99 & 139.28 & $1: 7.5: 0.3: 0.4: 0.7: 10.7$ \\
Hebei & 57.68 & 74.88 & 2.02 & 2.19 & 5.96 & 77.8 & $1: 1.2: 0.03: 0.03: 0.1: 1.3$ \\
\hline
\end{tabular}

Table 9: Recombination of SCC in Heibei MC

\begin{tabular}{ccccc}
\hline Groups & SOD(U/mL) & MDA(nmol/mL) & IL-6(ng/L) & MCP-1(ng/L) \\
\hline Control (BSA) & $6.56 \pm 0.59$ & $0.26 \pm 0.14$ & $3.15 \pm 0.17$ & $3.76 \pm 0.15$ \\
Model (AGEs) & $2.08 \pm 0.28^{\# \# \#}$ & $0.93 \pm 0.12^{\# \# \#}$ & $4.88 \pm 0.23^{\# \#}$ & $4.58 \pm 0.06^{\# \#}$ \\
Positive (AG) & $9.60 \pm 0.17^{* * *}$ & $0.39 \pm 0.04^{* * *}$ & $3.14 \pm 0.12^{* *}$ & $3.68 \pm 0.14^{* *}$ \\
Anhui & $7.71 \pm 0.98^{* *}$ & $0.46 \pm 0.05^{* *}$ & $3.20 \pm 0.11^{* *}$ & $3.67 \pm 0.12^{* *}$ \\
Hebei & $6.72 \pm 0.41^{*}$ & $0.55 \pm 0.01^{* * \$ \$}$ & $3.86 \pm 0.08^{* * \$ \$}$ & $4.31 \pm 0.09^{* \$ \$ \$}$ \\
Hebei recombination & $7.17 \pm 0.53^{* *}$ & $0.43 \pm 0.06^{* * \& \& \&}$ & $3.62 \pm 0.10^{* * \$ \$ \&}$ & $3.85 \pm 0.16^{* * \& \&}$ \\
\hline
\end{tabular}

Note: ${ }^{\# \#} P<0.01$ and ${ }^{\# \#} P<0.001$ vs. BSA group; ${ }^{*} P<0.05,{ }^{* *} P<0.01$ and ${ }^{* * *} P<0.001$ vs. AGEs group; ${ }^{\$} P<0.05,{ }^{\$} P<0.01$ and ${ }^{\$ \$} P<0.001$ vs. Anhui group; ${ }^{\&} P<0.05,{ }^{\& \&} P<0.01$ and ${ }^{\& \& \&} P<0.001$ vs. Hebei group.

six active components also have been reported to have a good therapeutic effect on kidney disease and diabetes $[14,15]$. In the present study, both SOD activity and MDA content of AGEs-induced mesangial cells were increased or decreased by active components screened from MC. The results indicated that the screened active components could inhibit oxidative stress of DN. IL-6 expression in mesangial cells was enhanced by the screened six active components of MC. The screened active components of MC could significantly down-regulate AGEs-induced $\mathrm{MCP}-1$. The selected components of $\mathrm{MC}$ also reduced $\mathrm{FN}$ secretion, down-regulated FN protein expression, which revealed that $\mathrm{MC}$ could inhibit the synthesis of FN to prevent the thickening of basement membrane.

In conclusion, the SCC of oxypaeoniflorin, paeoniflorin, trigalloyl glucose, pentagalloyl glucose, benzoylpaeoniflorin and paeonol in Heibei was $1: 1.2: 0.03: 0.03: 0.1: 1.3$, while the ratio with distinct pharmacodynamic effect in Anhui geoherb $\mathrm{MC}$ was 1:7.5:0.3:0.4:0.7:10.7. Hebei MC had worse efficacy and different SCC from Anhui MC. The results showed that the stable and orderly structural integrity of active components in $\mathrm{MC}$ from Anhui, geoherb one, acts as an important contributor to the prevention and treatment of DN. Adjusting SCC of Hebei MC (with worst efficacy) to geoherb MC from Anhui (with best efficacy) showed a similar activity in AGEs-induced HBZY-1 cells. These demonstrated that the efficacy of MC was related closely to its stable SCC.

This paper revealed the SCC of geoherb MC, initially provided the material basis for a better prevention and treatment of geoherb $\mathrm{MC}$ on $\mathrm{DN}$. The results demonstrated that the quality of $\mathrm{MC}$ materia medica was related closely to its stable SCC. Our findings provide evidence for the importance of SCC in CMM efficacy, and also provide a new insight into quality control of TCM.

\section{MATERIALS AND METHODS}

\section{Materials, solvent and chemicals}

Root bark of Paeonia suffruticosa Andr. were collected from 10 different regions including Tongling, Anhui province (Batch No. 20120415); Longnan, Gansu province (Batch No. 20120510); Dianjiang, Chongqing province (Batch No. 20120422); Heze, Shandong province (Batch No. 20120525); Guanxian, Sichuan province (Batch No. 20120603); Qingan, Zhejiang province (Batch No. 20120617); Yongzhou, Hunan province (Batch No. 20120622); Guiyang, Guizhou province (Batch No. 20120705); Baoding, Hebei province (Batch No. 20120711); Luoyang, Henan province (Batch No. 20120720), in October and November, 2011.

After excavation, MC was obtained by scraping and drying the root bark. The pharmacognosy identification of all the samples was identified by Professor Dekang $\mathrm{Wu}$ (Nanjing University of Chinese Medicine). The remaining voucher specimens were deposited at Jiangsu Provincial Academy of Chinese Medicine. The deposited number were No. ACM20120430 (Anhui), No. ACM20120518 (Gansu), No. ACM20120615 (Sichuan), No. ACM20120429 (Chongqing), No. ACM20120605 (Shandong), No. ACM20120628 (Zhejiang), No. ACM20120702 (Hunan), No. ACM20120717 (Guizhou), No. ACM20120726 (Hebei) and No. ACM20120806 (Henan), respectively.

SOD WST-1 assay kit (Lot: 20121101), MDA assay kit (Lot: 20121026), CAT assay kit (Lot: 20121011), GSH-Px assay kit (Lot: 20121018), and urinary protein kit (Lot: 20120923) were purchased from Jiancheng Bioengineering Institute (Nanjing, China). Rat IL-6 (Lot: 20130315) and MCP-1 (Lot: 20130311) ELISA kits were 
purchased from Victoria Reagent Co., Ltd. (Shanghai, China); RIPA cell lysates was offered by KeyGEN (Nanjing, China). Rabbit anti-mouse FN, TGF- $\beta 1$ and ICAM-1 monoclonal antibodies were offered by Boster Biological Engineering Co., Ltd. (Wuhan, China). Standard substances of oxypaeoniflorin, paeoniflorin, benzoylpaeoniflorin, paeonol, more than $98 \%$, were all ordered from National Instisutes for Food and Drug Control. Trigalloyl glucose, pentagalloyl glucose were supplied by BOC Sciences (NY, USA). Streptozotocin (STZ, Lot: 20130316) was offered by Sigma Aldrich (St. Louis., MO, USA). High-sugar high-fat diet was consisted of $10 \%$ sucrose, $10 \%$ egg yolk powder, $5 \%$ lard, $1 \%$ cholesterol and $0.2 \%$ bile salts $(\mathrm{W} / \mathrm{W})$.

\section{Sample preparations of MC from different regions}

MC from 10 different regions were processed as follows: $\mathrm{MC}$ of $1.0 \mathrm{~kg}$ was extracted with $8 \mathrm{~L}$ ethanol $(\mathrm{v} / \mathrm{v}, 75 \%)$ for three times $(2 \mathrm{~h} /$ time $)$ in reflux extraction device. The extracts were filtrated by gauze, merged and then concentrated to dry by rotavapor R-220 under reduced pressure (BUCHI, Switzerland). Then the dry powder was used for the further study.

\section{Preparation of AGEs}

The preparation of advanced glycation end products (AGEs) was as previous description [16]. Bovine serum albumin (BSA) of $5.0 \mathrm{~g}$ and D-glucose of $9.0 \mathrm{~g}$ were weighed accurately and then placed in $100 \mathrm{~mL}$ phosphate buffer solution ( $\mathrm{PBS}, \mathrm{pH}=7.2$ ) in sterile glass container. The solution was filtered over a $0.22 \mu \mathrm{m}$ membrane before being sealed and incubated three months at $37^{\circ} \mathrm{C}$. AGEs were obtained by the brown reaction solution being dialyzed to remove small molecules in PBS solution. A fluorescence spectrophotometer (PerkinElmer, Waltham, MA, USA) was used to measure AGEs-specific fluorescence at excitation/emission of $370 \mathrm{~nm} / 440 \mathrm{~nm}$. The content of AGEs was detected by AGEs ELISA kit, the final concentration was $858 \mathrm{mg} / \mathrm{mL}[17,18]$.

\section{Cell culture and treatment}

HBZY-1 mesangial cells were purchased from Shanghai Institute of Biochemistry and Cell Biology (Shanghai, China). HBZY-1 rat mesangial cells were maintained in low-glucose Dulbecco's modified Eagle's medium (DMEM) (Gibco, USA) supplemented with 10 $\%$ FBS (Gibco, USA). And cells were kept in an incubator with $5 \% \mathrm{CO}_{2}$ at $37^{\circ} \mathrm{C}$. Medium should be replaced every 2 days to.

After HBZY-1 cells in the logarithmic growth phase digestion, cells $\left(1.5 \times 10^{5}\right.$ cells/well $)$ were seeded in 24- well plates with $0.5 \mathrm{~mL}$ cell suspension per well. After being incubated for $24 \mathrm{~h}$, the medium was discarded. AGEs of $200 \mu \mathrm{g} / \mathrm{mL}$ was used to induce mesangial injury. Aminoguanidine (AG, $10 \mu \mathrm{M})$ was used as the positive control while BSA $(200 \mu \mathrm{g} / \mathrm{mL})$ as blank control. MC extract $(200 \mu \mathrm{g} / \mathrm{mL})$ was used to treat cells in the presence of $200 \mu \mathrm{g} / \mathrm{mL}$ AGEs. For active compounds, such as paeonol, trigalloyl glucose, pentagalloyl glucose, paeoniflorin, oxypaeoniflorin and benzoylpaeoniflorin, different concentrations $\left(10^{-4} \mathrm{M}, 10^{-5} \mathrm{M}\right.$, and $\left.10^{-6} \mathrm{M}\right)$ were used for evaluating activity. After treatment, cells were maintained in a HERA CELL 500 incubator (Thermo Scientific, MA, USA) with $5 \% \mathrm{CO}_{2}$ at $37^{\circ} \mathrm{C}$ for $24 \mathrm{~h}$.

\section{Animal model and drug treatment}

Male Sprague-Dawley rats (180-220 g), provided by Shanghai SLAC Laboratory Animal Co. Ltd. (Shanghai, China), were housed for $7 \mathrm{~d}$ to adapt the environment and maintained at a temperature of $25^{\circ} \mathrm{C}$ with the relative humidity of $45 \%$. All animals were housed in Experimental Animal Center in Jiangsu Province (Nanjing, China) on a $12 \mathrm{~h} / 12 \mathrm{~h}$ light/dark cycle with ad libitum access to food and water.

DN rats were developed according to previous method [8]. Ten rats were injected with $0.1 \mathrm{M}$ citrate buffer $(\mathrm{pH}=4.5)$ as blank control. Other rats were treated with the high glucose-fat diet for 2 weeks followed by a single intraperitoneal injection of $45 \mathrm{mg} / \mathrm{kg} \mathrm{STZ}$ (dissolved in $0.1 \mathrm{M}$ citrate buffer, $\mathrm{pH}=4.5$ ) for inducing $\mathrm{DN}$ rats. $\mathrm{DN}$ rats were confirmed successfully when blood glucose exceeded $16.7 \mathrm{mM}$, urine protein was superior to $20 \mathrm{mg} / 24$ h. DN rats were randomly divided into 12 groups with 10 rats in each group: the model group (DN), positive control group $(0.1 \mathrm{~g} / \mathrm{kg} \mathrm{AG}), \mathrm{MC}$ from 10 regions (5g crude drug/ $\mathrm{kg}$ ). The same amount of saline was used for control and model group. The administration was continued for 30 days. All animal experiment procedures were performed strictly in accordance with national and international laws for the use and care of laboratory animals.

After fasting, rats were weighed and anesthetized with $10 \%$ chloral hydrate $(3 \mathrm{~mL} / \mathrm{kg})$ by intraperitoneal injection. Rats were fixed to cut abdomen along the midline, $5 \mathrm{~mL}$ blood was taken from abdominal aortic. After being taken and weighted, kidney tissues were fixed with $10 \%$ formalin. The blood was centrifuged at $3000 \mathrm{rpm}$ for $10 \mathrm{~min}$. The supernatant was collected and stored at $-80^{\circ} \mathrm{C}$.

\section{Instrumentation and analysis conditions}

MC powder of $0.2 \mathrm{mg}$ was dissolved in $1 \mathrm{~mL}$ methanol with an ultrasound for $15 \mathrm{~min}$. Then the samples were centrifuged at $11000 \mathrm{rpm}$ for $10 \mathrm{~min}$. The supernatant was obtained for HPLC analysis.

Agilent (Palo Alto, CA, USA) 1100 series HPLC system, equipped with an online degasser, a quaternary 
pump, an auto-injector, a column thermostat and a DAD detector, was performed for analysis of components. The chromatographic experiments were conducted on Agilent TC-C ${ }_{18}$ column $(4.6 \times 250 \mathrm{~mm}, 5 \mu \mathrm{m})$ (Thermo Electron Corp, MA, USA) under gradient elution at $25^{\circ} \mathrm{C}$. Mobile phase was consisted of CAN (A) and $0.1 \%$ formic acid in water $(\mathrm{v} / \mathrm{v})(\mathrm{B})$. Elution gradient was as follows: 0-20 min, 5\%-10\%A; 20-30 min, 10\%-10\%A; 30-80 min, $10 \%-18 \% \mathrm{~A} ; 80-120 \mathrm{~min}, 18 \%-50 \% \mathrm{~A}$. The flow rate was set at $0.8 \mathrm{~mL} / \mathrm{min}$. The injection volume was $10 \mu \mathrm{L}$ and the UV detection wavelength was kept at $254 \mathrm{~nm}$.

Varian 310 triple quadrupole mass spectrometer equipped with ESI ion source (Varian, USA) was used for MS analysis in our study. Negative ion detection mode was conducted in this analysis. Nitrogen $\left(\mathrm{N}_{2}\right)$ was set at a flow rate of $0.45 \mathrm{~L} / \mathrm{min}$ as atomization gas, $0.2 \mathrm{~L} / \mathrm{min}$ as the curtain gas. The depolarization potential (DP) was -20 $\mathrm{V}$ while focusing potential was $-80 \mathrm{~V}$. The mass data was recorded within the range of 50-1200 Da.

\section{Assays for SOD, CAT, GSH-Px activity and MDA content}

Cell suspension or serum was centrifuged with 3000 rpm for $10 \mathrm{~min}$ and the supernatant was collected for the measurement. The levels of CAT, GSH-Px, SOD and MDA were determined according to manufacturer's protocols. Optical density (OD) value of samples was determined with microplate reader (Thermo Scientific, MA, USA) at wavelength of $405 \mathrm{~nm}$ (CAT), $412 \mathrm{~nm}$ (GSH-Px), $550 \mathrm{~nm}$ (SOD) and $532 \mathrm{~nm}$ (MDA) respectively.

\section{ELISA for inflammatory cytokines IL-6 and MCP-1}

Cell supernatant was taken for the determination of inflammatory cytokines. IL-6 and MCP-1 ELISA kits were used to determine cytokines levels according to manufacturer's protocols. Wavelength at $450 \mathrm{~nm}$ was chosen to read the OD value of samples with microplate reader (Thermo Scientific, MA, USA). The contents of inflammatory cytokines were calculated according to the standard curve.

\section{Western blotting analysis for TGF- $\beta 1$, ICAM-1 and FN}

HBZY-1 cells were washed with ice-cold PBS and cell lysate was performed on the ice for $30 \mathrm{~min}$. The lysates were centrifuged at $1500 \mathrm{rpm}$ for $3 \mathrm{~min}$, then supernatant was taken for measuring. An equal amount of total protein was separated by $10 \%$ SDS-PAGE and then transferred from the SDS-PAGE gel to PVDF membrane. After being blocked with $1 \%$ BSA in tris-buffered saline Tween-20 (TBST), membrane was incubated with the primary antibodies ICAM-1(1:400), TGF- $\beta 1$ (1:400), FN
(1:400) overnight. Then membrane was washed thrice and incubated with the peroxidase-conjugated secondary antibody (1:1000). Finally, the bands in the membrane incubated with chemiluminescence color and photographed by an ECL minicamera. $\beta$-actin was used as loading control. The quantification of proteins was analyzed by Image pro plus (IPP 6.0, Media Cybernetics, MD, USA) software.

\section{Immunohistochemistry for TGF- $\beta 1$ and ICAM-1 expressions}

Kidney tissues were dehydrated by serial gradient concentrations of alcohol, and then embedded in paraffin blocks for $3 \mathrm{~h}$. After the paraffin embedded, the tissues were cut into serial sections at the thickness of $5 \mu \mathrm{m}$. Sections were heated and washed with $10 \mathrm{mM}$ sodium citrate buffer. After quenching endogenous peroxidase and blocking with normal goat serum, the sections were incubated overnight at $4{ }^{\circ} \mathrm{C}$ with rabbit anti-rat antibodies specific against TGF- $\beta 1$ (1:200) and ICAM-1(1:200), respectively. After washing with $\mathrm{PBS}$, the sections were incubated at $37^{\circ} \mathrm{C}$ for $2 \mathrm{~h}$ with the secondary antibodies, and then stained by DAB. Finally, the results were observed under IX83 microscopic (Olympus, Japan).

\section{SOD activity, MDA, IL-6, MCP-1 content after component compatibility}

AGEs-induced HBZY-1 cell model was established as in the section "Cell culture and extract treatment", cells were divided into six groups. $200 \mu \mathrm{g} / \mathrm{mL}$ AGEs was used to induce mesangial injury. Aminoguanidine (AG) of $10 \mu \mathrm{M}$ was used as the positive control while BSA of $200 \mu \mathrm{g} / \mathrm{mL}$ as blank control. Drug groups were given extractions of MC $(200 \mu \mathrm{g} / \mathrm{mL})$ from Anhui, Hebei and Hebei transformations which the intrinsic ratio of oxypaeoniflorin, paeoniflorin, trigalloyl glucose, pentagalloyl glucose, benzoylpaeoniflorin and paeonol was adjusted from 1:1.2:0.03:0.03:0.1:1.3 to 1:7.5:0.3:0.4:0.7:10.7, respectively. Medium was added to $2 \mathrm{~mL}$ before culture plates were incubated at $37{ }^{\circ} \mathrm{C}$, $5 \% \mathrm{CO}_{2}$ for $24 \mathrm{~h}$. And then, SOD activity, MDA content and inflammatory cytokines like IL-6, MCP-1 were determined according to manufacturer's protocols.

\section{Data analysis}

All data were expressed as means \pm standard deviation (SD). Statistical analysis was performed by GraphPad Prism TM 5.0 statistical package with analysis of variance. Comparison between groups was using ANOVA, followed by Dunnett's significant post-hoc analysis for pair-wise multiple comparisons. The value of $P$ less than 0.05 was considered to be a statistically significant difference. Cluster analysis and principal component analysis were performed by SPSS 16.0. 


\section{Author contributions}

LF and JG analysis and interpretation of data, conception and design; MZ, CW and MZ acquisition of data and writing of the manuscript; GW, JS, XT, YZ, JC, $\mathrm{RL}, \mathrm{LQ}, \mathrm{CW}$ and $\mathrm{LZ}$, analysis and interpretation of data, statistical analysis; LF, XJ, development of methodology, revision of the manuscript, technical support, study supervision, revision of the manuscript.

\section{CONFLICTS OF INTEREST}

The authors declare that the research was conducted in the absence of any commercial or financial relationships that could be construed as a potential conflict of interest.

\section{FUNDING}

The authors gratefully acknowledge the financial support of the National Natural Science Foundation of China (81473394, 81503314, 81703775); Significant changes in the central level support projects (2060302); "333 high-level personnel training project" of Jiangsu Province (BRA2015475); “Six Talent Peak" Project of Jiangsu Province (YY-012); Innovative Research Team of Health Development Project with Science and Education in Jiangsu Province (CXTDB2017003); Program for Innovative Research Team of Six Talent Peaks Project in Jiangsu Province (SWYY-CXTD-004); Scientific Research Project of State Traditional Chinese Medicine Clinical Base Construction (JDZX2015072) and Jiangsu Province Youth Medical Key Talent Project (QNRC2016634) and Jiangsu province high-level health personnel "Five-One Project " (LGY2017085).

\section{REFERENCES}

1. Huang L, Guo L, Ma C, Gao W, Yuan Q. Top-geoherbs of traditional Chinese medicine: common traits, quality characteristics and formation. Front Med. 2011; 5:185-194.

2. Xiao X, Wang J, Yan D, Lv Y. [Establishment of Dao-Di index and its significance in quality control and rational usage of Chinese medicine]. [Article in Chinese]. Zhongguo Zhong Yao Za Zhi. 2012; 37:1513-16.

3. Zhang MH, Feng L, Hu SY, Jia XB. [Essence of material base in geoherbs: specificality of constituent structure]. [Article in Chinese]. Zhongguo Zhong Yao Za Zhi. 2013; 38:136-40.

4. Dan H, Zhang L, Qin X, Peng X, Wong M, Tan X, Yu S, Fang N. Moutan cortex extract exerts protective effects in a rat model of cardiac ischemia/reperfusion. Can J Physiol Pharmacol. 2016; 94:245-250.

5. Qiu H, Zhang L, Zhu M, Zhang M, Chen J, Feng L, Jia X, Jacob JA. Capture of anti-coagulant active ingredients from Moutan Cortex by platelet immobilized chromatography and evaluation of anticoagulant activity in rats. Biomed Pharmacother. 2017; 95:235-244.

6. Behrendt P, Perin P, Menzel N, Banda D, Pfaender S, Alves MP, Thiel V, Meulemann P, Colpitts CC, Schang LM, Vondran FW, Anggakusuma, Manns MP, et al. Pentagalloylglucose, a highly bioavailable polyphenolic compound present in Cortex moutan, efficiently blocks hepatitis C virus entry. Antiviral Res. 2017; 147:19-28.

7. Zhang MH, Feng L, Zhu MM, Gu JF, Jiang J, Cheng XD, Ding SM, Wu C, Jia XB. The anti-inflammation effect of Moutan Cortex on advanced glycation end productsinduced rat mesangial cells dysfunction and High-glucosefat diet and streptozotocin-induced diabetic nephropathy rats. J Ethnopharmacol. 2014; 151:591-600.

8. Jang MH, Kim KY, Song PH, Baek SY, Seo HL, Lee EH, Lee SG, Park KI, Ahn SC, Kim SC, Kim YW. Moutan Cortex Protects Hepatocytes against Oxidative Injury through AMP-Activated Protein Kinase Pathway. Biol Pharm Bull. 2017; 40:797-806.

9. Zhang M, Feng L, Gu J, Ma L, Qin D, Wu C, Jia X. The attenuation of Moutan Cortex on oxidative stress for renal injury in AGEs-induced mesangial cell dysfunction and streptozotocin-induced diabetic nephropathy rats. Oxid Med Cell Longev. 2014; 2014:463815.

10. Chen J, Zhang L, Zhang MH, Zhao D, Yuan JR, Feng L, Jia XB. [Inhibitory effects of extracts from Moutan Cortex on formation of advanced glycation end-products (AGEs) in vitroand specificity screening of its potential active components]. [Article in Chinese]. Zhongguo Zhong Yao Za Zhi. 2016; 41:891-97.

11. Chen J, Hou XF, Wang G, Zhong QX, Liu Y, Qiu HH, Yang N, Gu JF, Wang CF, Zhang L, Song J, Huang LQ, Jia XB, et al. Terpene glycoside component from Moutan Cortex ameliorates diabetic nephropathy by regulating endoplasmic reticulum stress-related inflammatory responses. J Ethnopharmacol. 2016; 193:433-444.

12. Ha do T, Ngoc TM, Lee I, Lee YM, Kim JS, Jung H, Lee S, $\mathrm{Na} \mathrm{M}$, Bae K. Inhibitors of aldose reductase and formation of advanced glycation end-products in moutan cortex (Paeonia suffruticosa). J Nat Prod. 2009; 72:1465-1470.

13. Shi JY, Yuan XF, Lin HR, Yang YQ, Li ZY. Differences in soil properties and bacterial communities between the rhizosphere and bulk soil and among different production areas of the medicinal plant Fritillaria thunbergii. Int J Mol Sci. 2011; 12:3770-3785.

14. Zhang $\mathrm{MH}$, Feng $\mathrm{L}$, Zhu MM, Gu JF, Wu C, Jia XB. Antioxidative and anti-inflammatory activities of paeoniflorin and oxypaeoniflora on AGEs-induced mesangial cell damage. Planta Med. 2013; 79:1319-1323.

15. Lee H, Lee G, Kim H, Bae H. Paeonol, a major compound of moutan cortex, attenuates Cisplatin-induced nephrotoxicity in mice. Evid Based Complement Alternat Med. 2013; 2013:310989.

16. Li Y, Liu S, Zhang Z, Xu Q, Xie F, Wang J, Ping S, Li C, Wang Z, Zhang M, Huang J, Chen D, Hu L, et al. RAGE 
mediates accelerated diabetic vein graft atherosclerosis induced by combined mechanical stress and AGEs via synergistic ERK activation. PLoS One. 2012; 7:e35016.

17. Feng $\mathrm{L}$, Zhu M, Zhang M, Gu J, Jia X, Tan X, Gao C, Zhu Q. The protection of 4,4'-diphenylmethanebis(methyl) carbamate from Cortex Mori on advanced glycation end product-induced endothelial dysfunction: via inhibiting AGE formation or blocking AGEs-RAGE axis? Fitoterapia. 2013; 89:239-249.
18. Tan X, Gu J, Zhao B, Wang S, Yuan J, Wang C, Chen J, Liu J, Feng L, Jia X. Ginseng improves cognitive deficit via the RAGE/NF-kB pathway in advanced glycation end productinduced rats. J Ginseng Res. 2015; 39:116-124. 